\title{
A RENORMING OF NONREFLEXIVE BANACH SPACES
}

\author{
WILLIAM J. DAVIS AND WILLIAM B. JOHNSON ${ }^{1}$
}

ABSTraCt. Every nonreflexive Banach space can be equivalently renormed in such a way that it is not isometrically a conjugate space.

Dixmier [1] asked: "If $X$ is isomorphic to a conjugate Banach space, is $X$ isometric to a conjugate space?" Klee [5] gave a negative solution by giving an equivalent norm for $l^{\infty}$ under which that space is not isometrically a dual space. Here, we show that such a norm exists for every nonreflexive Banach space. The result is precise since, obviously, if $X$ is reflexive, it is isometrically a conjugate space under any equivalent renorming.

The prototype of our first lemma is the renorming theorem of Kadec ([2], [3]) and Klee [6]. The version we give may have some novelty since we do not assume separability of $X$. We feel that the proof we sketch here is somewhat more revealing than existing proofs of the Kadeć-Klee theorem (cf. [4], [7] and [8, p. 486]).

Lemma 1. Suppose $(X,\|\cdot\|)$ is a Banach space, $Y$ is a separable closed subspace of $X^{*}$, and that $Z$ is a subspace of $X$ which is norm determining over $Y$ (that is, $\sup \{y(z): z \in Z,\|z\|<1\}=\|y\|$ for all $y \in Y)$. Then, there is an equivalent norm, $\|\cdot\| \cdot \|$, on $X$ such that, if $\left(x_{\delta}^{*}\right)$ is a net in $X^{*}, y \in Y$, $x_{\delta}^{*}(z) \rightarrow y(z)$ for each $z \in Z$ and $\left\|x_{\delta}^{*}\right\| \rightarrow\|\| y \|$, then $\left\|x_{\delta}^{*}-y\right\| \rightarrow 0$.

SKETCH OF PROOF. Let $E_{1} \subset E_{2} \subset \cdots$ be a sequence of finite dimensional subspaces of $Y$ with $\bigcup E_{n}$ dense in $Y$. For each $x^{*}$ in $X^{*}$, define $\left\|x^{*}\right\|=$ $\left\|x^{*}\right\|+\sum 2^{-n} d\left(x^{*}, E_{n}\right)$, where $d\left(x^{*}, E_{n}\right)$ is the usual distance from $x^{*}$ to $E_{n}$. It is readily verified that each of the functions $x^{*} \rightarrow d\left(x^{*}, E_{n}\right)$ is weak* lower semicontinuous. It follows that the ball in $\left(X^{*},\|\cdot \cdot\|\right)$ is weak* closed, so that $\|\cdot|\||$ is the dual norm for some norm $\| \cdot\|\cdot\|$ on $X$, equivalent to $\|\cdot\|$.

Suppose that $\left(x_{\delta}^{*}\right) \subset X^{*}, y \in Y$ are as in the statement of the lemma. Since $Z$ is norm determining over $Y$, one observes that $\lim \inf _{\delta}\left\|x_{\delta}^{*}\right\| \geqq\|y\|$ and, for each $n$,

$$
\lim _{\delta} \inf d\left(x_{\delta}^{*}, E_{n}\right) \geqq d\left(y, E_{n}\right),
$$

Received by the editors May 6, 1972.

AMS (MOS) subject classifications (1970). Primary 46B10, 46B15.

Key words and phrases. Nonreflexive space, conjugate space, dual space.

1 The second author was supported in part by NSF GP-28719. 
so that in fact

$$
\lim _{\delta} d\left(x_{\delta}^{*}, E_{n}\right)=d\left(y, E_{n}\right) \text {. }
$$

Since $d\left(y, E_{n}\right) \rightarrow 0$, one has $\left\|x_{\delta}^{*}-y\right\| \rightarrow 0$.

Lemma 2. If $X$ is a nonreflexive Banach space, then $X^{*}$ contains a separable subspace $Y$ such that the natural map $T: X \rightarrow Y^{*}$ (defined by $(T x)(y)=y(x)$ for all $x, y)$ is not onto $Y^{*}$.

Proof. By Eberlein's theorem, the ball of $X^{*}$ is not weakly countably compact, so there is a countable net $\left(x_{\delta}^{*}\right)$ in the ball of $X^{*}$ which has no weakly convergent subnet. Let $\left(x_{\lambda}^{*}\right)$ be a subnet which converges weak* to $y$ in $X^{*}$. Let $Y$ be the closed linear span of $\left(x_{\delta}^{*}\right) \cup(y)$. For each $x$ in $X$, $(T x)\left(x_{\lambda}^{*}\right) \rightarrow(T x)(y)$, but $y^{*}\left(x_{\lambda}^{*}\right)$ fails to converge to $y^{*}(y)$ for some $y^{*}$ in $Y^{*}$ so $y^{*} \notin T X$.

THEOREM. If $(X,\|\cdot\|)$ is a nonreflexive Banach space, there is an equivalent norm, $\|\cdot\| \cdot \|$, on $X$ under which $X$ fails to be isometric to a conjugate space.

Proof. Let $Y$ be the subspace of $X^{*}$ from Lemma 2, $Z=X$ and $\|\cdot \cdot\|$ the norm of Lemma 1. If $(X,\|\cdot \cdot\| l)$ is isometric to $W^{*}$, then consider $W$ as being canonically embedded in $X^{*}\left(=W^{* *}\right)$. Since the ball of $W$ is weak* dense in that of $W^{* *}$, for each $y$ in $Y$, there is a net $\left(w_{\delta}\right)$ in $W$ with $\left\|w_{\delta}\right\| ! \leqq\|y\|$ for all $\delta$ and $w_{\delta}(x) \rightarrow y(x)$ for each $x$ in $X$. Since lim inf $\left\|w_{\delta}\right\| \geqq$ $\|y\|\|,\| w_{\delta}\|\| \rightarrow\left\|y_{i}\right\|$ so, by Lemma $1, w_{\delta} \rightarrow y$. Since $W$ is a closed subspace, $Y \subset W$. However, the natural map of $X$ to $W^{*}$ must be onto which forces the natural map of $X$ to $Y^{*}$ to be onto. This contradicts the choice of $Y$, completing the proof.

We recall that $X$ is isometric to a conjugate space if and only if there is a norm one projection $P$ of $X^{* *}$ onto $X$ such that $(I-P) X^{* *}$ is weak* closed [1]. The weak* closedness of $(I-P) X^{* *}$ is essential (viz., $\left.L_{1}([0,1])\right)$. This raises the problem: Can every nonreflexive space be renormed so that it is not the range of a norm one projection on its second conjugate space?

\section{REFERENCES}

1. J. Dixmier, Sur un théorème de Banach, Duke Math J. 15 (1948), 1057-1071. MR 10, 306.

2. M. I. Kadeć, On the connection between weak and strong convergence, Dopovidi Akad. Nauk Ukraïn. RSR 1959, 949-952. (Ukrainian) MR 22 \#2879.

3. - Topological equivalence of all separable Banach spaces, Dokl. Akad. Nauk SSSR 167 (1966), 23-25=Soviet Math. Dokl. 7 (1966), 319-322. MR 34 \#1828.

4. M. I. Kadeć and A. Pelczyński, Basic sequences, bi-orthogonal systems and norming sets in Banach and Fréchet spaces, Studia Math. 25 (1965), 297-323. (Russian) MR 31 \#6112. 
5. V. L. Klee, Jr., Some characterizations of reflexivity, Revista Ci. Lima 52 (1950), nos. 3-4, 15-23. MR 13, 250.

6. - Mappings into normed linear spaces, Fund. Math. 49 (1960/61), 25-34. MR 23 \#A3985.

7. J. Rainwater, On a renorming theorem of Klee (preprint).

8. I. Singer, Bases in Banach spaces. I, Die Grundlehren der math. Wissenschaften, Band 154, Springer-Verlag, Berlin and New York, 1970.

Department of Mathematics, Ohio State University, Columbus, Ohio 43210 